University of Nebraska - Lincoln

DigitalCommons@University of Nebraska - Lincoln

$11-2021$

\title{
Strategies for highlighting items within visual scene displays to support augmentative and alternative communication access for those with physical impairments
}

Kevin Pitt

John W. McCarthy

Follow this and additional works at: https://digitalcommons.unl.edu/specedfacpub

Part of the Accessibility Commons, and the Special Education and Teaching Commons

This Article is brought to you for free and open access by the Department of Special Education and Communication Disorders at DigitalCommons@University of Nebraska - Lincoln. It has been accepted for inclusion in Special Education and Communication Disorders Faculty Publications by an authorized administrator of DigitalCommons@University of Nebraska - Lincoln. 


\title{
Strategies for highlighting items within visual scene displays to support augmentative and alternative communication access for those with physical impairments
}

\author{
Kevin M. Pitt ${ }^{1}$ and John. W. McCarthy ${ }^{2}$ \\ 1 Department of Special Education and Communication Disorders, \\ University of Nebraska-Lincoln, Lincoln, NE \\ 2 Division of Communication Sciences and Disorders, \\ Ohio University, Athens, $\mathrm{OH}$ \\ ORCID \\ Kevin M. Pitt http://orcid.org/0000-0003-3165-4093 \\ John. W. McCarthy http://orcid.org/0000-0001-6356-4266
}

\begin{abstract}
Purpose: In contrast to the traditional grid-based display, visual scene displays (VSDs) offer a new paradigm for aided communication. For individuals who cannot select items from an AAC display by direct selection due to physical impairments, AAC access can be supported via methods such as item scanning. Item scanning sequentially highlights items on a display until the individual signals for selection. How items are highlighted or scanned for AAC access can impact performance outcomes. Further, the effectiveness of a VSD interface may be enhanced through consultation with experts in visual communication. Therefore, to support AAC access for those with physical impairments, the aim of this study
\end{abstract}

Published in Disability and Rehabilitation: Assistive Technology, 2021

doi:10.1080/17483107.2021.2003455

Copyright (c) 2021 Informa UK Limited, trading as Taylor \& Francis Group. Used by permission.

Submitted 25 May 2021; revised 8 September 2021; accepted 2 November 2021; published 17 November 2021.

Supplemental data for this article is attached to the archive record page. 
was to evaluate the perspectives of experts in visual communication regarding effective methods for highlighting VSD elements.

Methods: Thirteen participants with expertise related to visual communication (e.g., photographers, artists) completed semi-structured interviews regarding techniques for item highlighting.

Results: Study findings identified four main themes to inform how AAC items may be highlighted or scanned, including (1) use of contrast related to light and dark, (2) use of contrast as it relates to color, (3) outline highlighting, and (4) use of scale and motion.

Conclusion: By identifying how compositional techniques can be utilized to highlight VSD elements, study findings may inform current practice for scanningbased AAC access, along with other selection techniques where feedback or highlighting is used (e.g., eye-gaze, brain-computer interface). Further, avenues for just-in-time programming are discussed to support effective implementation for those with physical impairments.

\section{Implications for rehabilitation}

- Findings identify multiple potential techniques to improve scanning through items in a photograph for individuals with severe motor impairments using alternative access strategies.

- Study findings inform current practice for scanning-based AAC access, along with other selection techniques where feedback or highlighting is used (e.g., eyegaze, brain-computer interface).

- Avenues for just in time programming of AAC displays are discussed to decrease programming demands and support effective implementation of study findings.

Keywords: Augmentative and alternative communication, AAC, visual scene displays, scanning; highlighting; artificial intelligence, just-in-time programming, cerebral palsy

The approximately ninety-seven million individuals worldwide who cannot use their natural speech to meet their daily communication needs represent an extremely heterogeneous group with a large variety of needs and skills [1-3]. This diverse group requires a range of tools and benefits from new developments and directions in technology. Advances in technology can open new possibilities in terms of communication, but there are new challenges when a system must be accessed in a different way from the original design. For example, touchscreen displays offer an immediate connection with the item seen and selected. However, for individuals with severe physical impairments, the touchscreen display may not be accessed via touch [4]. 
When motor access is not the primary driver of a technology innovation, the allowance for different kinds of AAC access techniques may be considered, depending upon the individual's profile and environment [5]. Sometimes access extensions are a logical and "small-step adaptation," and in other cases there are multiple significant pieces to consider.

Visual scene displays (VSDs) are a more recent innovation that have offered a new paradigm for aided communication displays [1,2]. Traditionally, items for AAC selection are presented in isolation on a plain background, without context, commonly arranged within a grid by taxonomic category (e.g., a grid of different animals) [6]. In comparison to traditional grid-based displays, VSDs may be more limited in the number of communication options provided at any one time. This restriction is because VSDs are limited to item choices that naturally occur within the scene. However, VSDs present symbols in naturally occurring context (e.g., within a photograph), with an intuitive navigation system based on the objects natural location in the scene itself $[2,7]$. Furthermore, just-in-time (JIT) programing aims to support the individuals' communication access and language learning in the moment to bolster communication success [8]. Positively, VSDs can be generated JIT, meaning that they are easily captured via an embedded digital camera and elements of the scene are then designated as communicative hotspots [9]. The easy process helps decrease the programmer demands to build displays for the individuals that use VSDs, possibly leading to greater engagement [10]. Therefore, these described advantages mean VSDs offer several benefits to traditional grid-based displays for children in the early symbolic stages of development [11] or adults with diagnosis such as aphasia [12]. Thus, it is important to consider how VSDs may be designed to support communication access for those with severe physical impairments.

For individuals who cannot directly select items from an AAC interface due to severe physical impairments, AAC access can be supported via item scanning. During scanning-based AAC access, items on a display are sequentially presented with the user signaling selection when their target item is offered. For instance, items may be presented through a visual box or outline or by an auditory signal, with the individual making an item selection by pressing a switch. However, scanning methods can significantly slow down communication 
rate relative to direct selection [13]. Further, changes to scanning patterns (e.g., highlighting each item singly from left to right and top to bottom, highlighting rows and then items in each column of that row) may also create differential problems with accuracy and efficiency [14]. In contrast to grid-based AAC displays, hotspots in VSDs follow the characters and items in an interactive scene and are generally not offered in neat rows and columns or on a plain background. Thus, supporting VSD access for those with severe physical impairments by scanning techniques may not be as simple as merely utilizing traditional, grid-based scanning approaches.

Light and Lindsay [15] described scanning as involving an offer (the highlighted items), selection, and then feedback on selection of an item. Recognizing the challenge of using scanning as an access method, Light and Lindsay [15] suggested that scanning could be improved to reduce the learning demands for young children by changing the teaching method/metaphor to describe the technique; or changing the technology to create more intuitive item offers and feedback, changing the nature of the cursor to clearly indicate which item was being offered as a choice, and clarifying the feedback given once the switch was activated to select an item (i.e., the offer/feedback). Teaching techniques for scanning continue to be effective, with metaphors tailored to match the graphic display scheme. For instance, using a metaphor like "playing tag" would be needed to help clarify the function of a travelling border cursor for young children. However, although effective teaching is important, teaching strategies are not the only consideration for supporting AAC access via item scanning, with adaptions to the visual interface also bolstering AAC success $[1,16]$.

McCarthy et al. [16] adapted the visual AAC interface by replacing the traditional cursor with rapidly enlarging symbols to in presenting communication items (i.e., a row of animals) to 2- to 3-year-old children without disabilities. The effect was to have an actual "offer" of the animals so that the enlarging images would simulate something being held closer to the user. The authors also added a feedback animation where the selected animal would travel to the middle of the screen and grow even larger in size to clearly demonstrate selection feedback. The study provided evidence that only under the enhanced scanning condition did children significantly improve their 
performance from baseline. However, these results were still part of a grid-based model. The idea of extending the work to visual scenes created challenges related to potentially overlapping items and a dilution of the scale effect when items within a scene are naturally of different sizes $[2,16]$.

In 2017, McCarthy and Boster [1] explored animating items within a line-drawn scene of a living room to innovate scanning for VSDs. Rather than enlarging items as a highlighting technique, the items were offered through animation, during which an item moved as it would normally function (e.g., the hands on a clock would move around its face, the curtains would blow in the wind, the light would turn on and off). The enhanced scanning was compared to a green border outline of highlighted items in the display. On average, the 2.5- to 3.5-year-old children showed greater improvement using the enhanced scanning method. Although this was a new attempt in exploring enhanced scanning in VSDs, there were several limitations. The items were only in a line-drawn room and, unlike what is suggested in VSD best practice [10], there were no people in the room or action routines represented. Finally, the programming of the room and the animations was significant. Therefore, although capturing digital images and movies is easier with current mobile technologies, answers to questions about enhancing scanning in evidence-based VSDs remain.

VSDs are meant to map onto and enhance participation in realworld interactions and routines. Therefore, the extent to which a VSD draws/demands a user's attention over time is an important consideration [5]. Scanning requires more continuous monitoring of a VSD, and repeated viewing of accompanying movies/animations may add to the monitoring time. Considering the visual attention patterns of individuals who use AAC may inform AAC design and support positive communication outcomes [17]. Therefore, the effectiveness of a VSD interface may be enhanced through consultation with multidisciplinary specialists, such as those with expertise in visual-cognitive sciences $[13,18]$ and visual communication, such as photographers [2]. For instance, based on interviews with visual communication experts, Pitt \& McCarthy [2] recently identified how principles of composition such as contrast, structure, shape and space, line, scale, and focus could be applied to help a) lower VSD complexity, b) help objects 
strand out from their surroundings, c) draw attention to key scene elements, and d) support selection of related scene vocabulary. However, the general composition guidelines presented by Pitt \& McCarthy [2] were largely focused on supporting VSD use by adults and children without severe physical impairments due to diagnosis such as intellectual disability [19], and aphasia [7]. In more detail, some individuals without severe physical impairments may access a VSD hotspot by touch selection. Further, some individuals with aphasia may not require item hotspots, but instead use VSD supports as shared communication tools to strengthen understanding of related scene vocabulary [7]. Neither of these AAC strategies require item highlighting for VSD use in a manner similar to that of scanning-based communication access. Therefore, how principles of visual composition can be utilized to enhance the offer of specific items within VSD during item highlighting to support access for those with severe physical impairments is unclear. Exploring effective offers is critical to support AAC access for those with severe physical impairments, even beyond scanning-based AAC designs. For instance, eye-tracking selection methods still often provide visual feedback regarding where an individual's gaze is currently located. Likewise, highlighting is important in P300based brain-computer interface $(\mathrm{BCl})$ paradigms, so a clear understanding of effective highlighting to build strong P300 responses is critical [20-22]. Therefore, to inform AAC design and support VSD access for those with severe physical impairments, the aim of this study was to evaluate how composition principles could be applied to help draw attention to focal scene elements during item highlighting to inform AAC design.

\section{Methods}

\section{Participants}

The participants in this study were part of a larger set of interviews done to investigate perspectives related to VSD composition for AAC techniques that do not use item highlighting [2]. Institutional Review Board approval was granted by the University of Nebraska-Lincoln and Ohio University. Participants with a wide range of experience 
related to photographic/artistic composition were recruited. The thirteen participants ( 7 females; $M=42.5$ years, $S D=9.8$, range $=23-55$ ) had an average of 17.5 years $(S D=10.7$, range $=4-38)$ experience. Experts needed to have a minimum of 2 years' experience related to composition, report feeling comfortable discussing composition principles, and report the ability to extend their knowledge to AAC photos and scanning. To ensure all participants had a common frame of reference, a short presentation about VSDs was shown prior to conducting the interviews. The presentation included a general description about the field of AAC, VSDs, hot spots, and AAC scanning. A link to a public video showing VSD use was provided for reference, along with images from McCarthy and Boster [1] to demonstrate scanningbased access to contextual scenes. The video was posted on an AACfocused university's webpage and included scenes of a trip that both included and excluded people. Table 1 includes a summary of the demographic information of the participants. Participants had minimal experience with individuals with disabilities, although one participant had used a camera to teach about self-expression to their brother diagnosed with Down syndrome.

Table 1. Participant information.

\begin{tabular}{lllll} 
Participant & Age & Gender & $\begin{array}{l}\text { Years of } \\
\text { Experiences }\end{array}$ & Primary Area of Expertise \\
\hline P1 & 38 & F & 21 & Photography \\
P2 & 48 & M & 10 & Light use in Composition \\
P3 & 49 & F & 23 & Photography/Film making \\
P4 & 31 & M & 8 & Photography, Drawing, Printmaking \\
P5 & 50 & F & 32 & Photography \\
P6 & 42 & M & 14 & Human attention during scene perception \\
P7 & 30 & F & 15 & Photography \\
P8 & 55 & M & 38 & Visual Art \\
P9 & 23 & F & 5 & Journalism/ Photography \\
P10 & 43 & M & 13 & Cinematography \\
P11 & 50 & F & 4 & Commercial Photography \\
P12 & 53 & M & 30 & Visual Photography \\
P13 & 40 & F & 14 & Film \\
\hline
\end{tabular}

Figure taken from Pitt and McCarthy [2]. Reprinted with permission from ASHA Journals. 


\section{Materials}

The interview guide (see Supplemental material A) was developed to fill gaps in current literature regarding how compositional factors (e.g., light and color contrast, scale) can be applied to highlight scene elements. Results from five additional interview questions discussing the application of composition principles to general scene design are reported in Pitt and McCarthy [2].

\section{Procedures}

Interviews lasted approximately $45 \mathrm{~min}$ and were completed in one session via either video conference $(n=12)$, or telephone $(n=1)$. The first author completed all interviews, and a script was used to ensure consistency and prevent any interviewer drift [23]. The interviewer reviewed the VSD background presentation with each participant and answered any related questions prior to starting the scripted questions. The interviewer asked follow-up questions and requested clarification when needed for accuracy. Some participants clarified by sharing personal or web-based images to support communication about composition principles. The interviewer also took notes to prompt follow-up questions and support later analysis. Notes also included key phrases to quote or that seemed to indicate a potential thematic area. The number of participants to be included was driven by a determination of saturation [24]. Specifically, when all members of the research team (including two SLPs, one with a degree in photography and video and one with experience in photography, plus one graduate student in speech-language pathology with a background in visual processing) agreed that collecting new data no longer revealed new insights or broader thematic areas and when topics had been discussed in sufficient detail [24]. Agreement was quantified by the research team stating that data collection would stop when no new codes regarding highlighting strategies were identified for six consecutive participants, and when the team found the themes were fully comprehensible with $100 \%$ consensus. 


\section{Data analysis}

A graduate assistant transcribed all interviews exactly as recorded (including pauses and non-linguistic content). Interview transcriptions were checked against the script for procedural reliability and accuracy by a second graduate assistant with discrepancies discussed to $100 \%$ consensus. NVivo software [25] was used for organization and analysis. Themes emerged using NVivo's coding features by constantly incorporating incoming data into the existing coding structure, and accommodating new data by adding codes $[26,27]$. Following coding, a codebook was developed detailing the four themes and seven subthemes identified (Supplementary material B). The first author and a graduate assistant re-evaluated the transcripts with the codebook for an initial pass at consistency. Discrepancies with the codebook and transcripts were discussed among the team until achievement of consensus. For reliability, 23\% (3/13) of the interview transcripts were coded separately by a trained research assistant using the codebook. Based upon the procedures of O'Neill and Wilkinson [28] and Pitt and McCarthy [2], percent agreement [29] was chosen for evaluating reliability for the randomly identified transcripts. A graduate assistant was used for intercoder reliability with training, guided practice, and then independent verification, until more than $80 \%$ accuracy was achieved at the level of the subtheme [28,30]. For our investigation, intercoder reliability was $100 \%$ for each transcript.

\section{Credibility indicators}

Additional checks on the data for quality and representativeness included member checking, peer debriefing and review, and triangulation $[30,31]$.

\section{Member checking}

During the interview, member checking was completed by the interviewer by 1) requesting clarification of any unclear statements during the interview process based on field notes, and 2) offering summary statements as checks for understanding of key points during the interview. For more deliberative consideration, a discussion summary was sent to each interviewee after the interview so they could confirm 
that their ideas were adequately represented in our evaluation. All of those who responded (12/13) indicated agreement with the summaries as sent. No concern was noted for the single participant who did not return their member check since multiple procedures for data credibility were utilized, and participant statements were consistent with prior interviewees.

Peer debriefing, review, and triangulation

The second author provided peer review of study methods across the length of the investigation. In addition, to confirm study findings were consistent with current theories regarding visual composition, following data analysis, a peer briefing procedure was completed [31]. The peer debriefer, who is currently completing their $\mathrm{PhD}$ in media communications, had 15 years' experience related to composition. The peer debriefer reviewed a copy of the codebook and discussed study findings with the lead author before indicating that study findings were consistent with current theories regarding visual composition based on their expertise. Finally, using a team approach helped decrease the possibility of lead author bias.

\section{Procedural reliability}

All procedures were completed with $98 \%$ accuracy as one participant (P11) indicated they did not receive the VSD presentation prior to beginning the interview. In this case, the interview, which used videoconferencing, only continued after the presentation was discussed, all the participant's questions were answered, and they indicated they still wished to continue with the interview. Procedural reliability was tracked via spreadsheet for study procedures related to transcription, distribution of the VSD presentation, and distribution of participant member check documents.

\section{Results}

Table 2 provides a summary of identified themes and subthemes, with the full codebook provided in Supplementary material B. The following results section is structured by theme and subtheme. 
Table 2. Identified themes, and subthemes.

\begin{tabular}{ll} 
Themes & Subthemes \\
\hline Use of contrast (light and dark) & $\begin{array}{l}\text { Make background darker and item lighter. } \\
\text { Make background of object brighter creating a halo } \\
\text { like effect around the item, possibly during a puls- } \\
\text { ing flash. }\end{array}$ \\
$\begin{array}{l}\text { Saturate target item color and desaturate background. } \\
\text { Keep the items natural color and make background } \\
\text { black and white. } \\
\text { Place a non-natural color overlay on object (e.g., a } \\
\text { semi-transparent green shape). } \\
\text { Outline highlighting in color, possibly with a more } \\
\text { muted colored background, though we need to } \\
\text { consider individuals color vision. }\end{array}$ \\
Outline Highlighting \\
Zooming movement and Scale.
\end{tabular}

\section{Use of contrast (light and dark)}

A total of nine experts described how light contrast may be utilized to draw attention to focal elements during item highlighting. In further detail, nine participants discussed the item of interest could be made brighter relative to the background, similar to a spotlight, drop shadow or glisten effect (Figure 1). In addition, the whole scene may fade to black with the focal object staying lit. For instance, P4 noted,

"It's highlighted and then everything else sort of either darkens or fades, uh because then you're removing sort of a distraction um from the rest of the image."

In addition, P9 noted

"I think people, like humans in general, have always directed our attention on theaters, for example, with a spotlight and so even having like a very obvious spotlight that you're moving around could be something to do."

In contrast to making the focal item brighter, five participants discussed how making the background brighter relative to the item of 


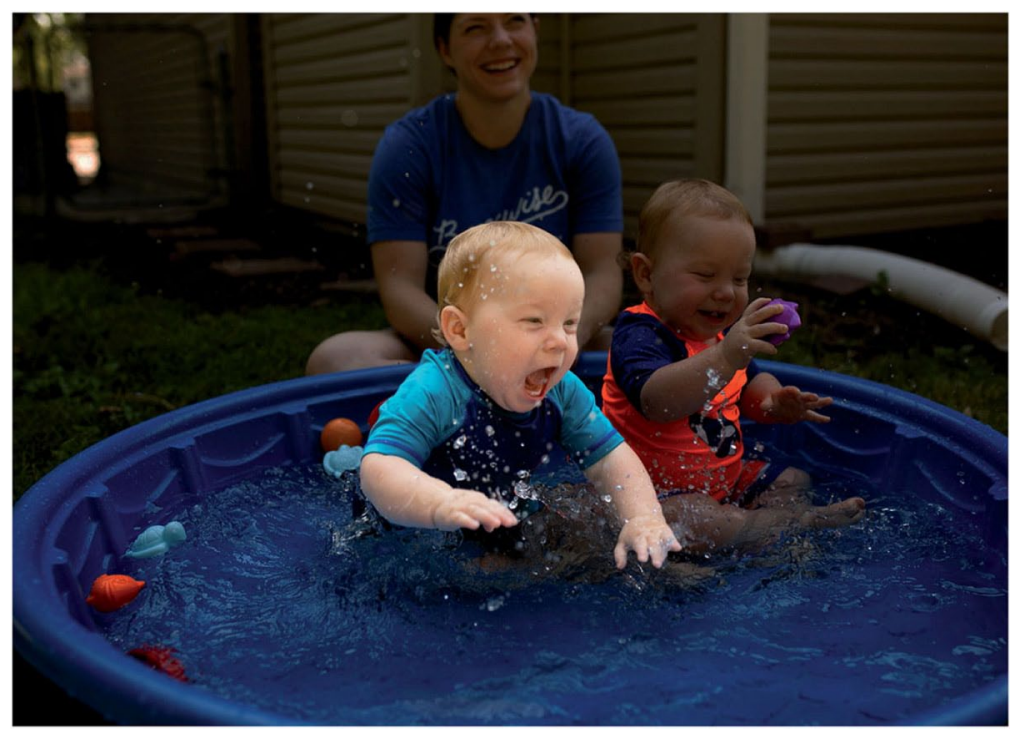

Figure 1. A visual scene display image including two children engaged in pool play. The central child is highlighted through increasing brightness in relation to the background.

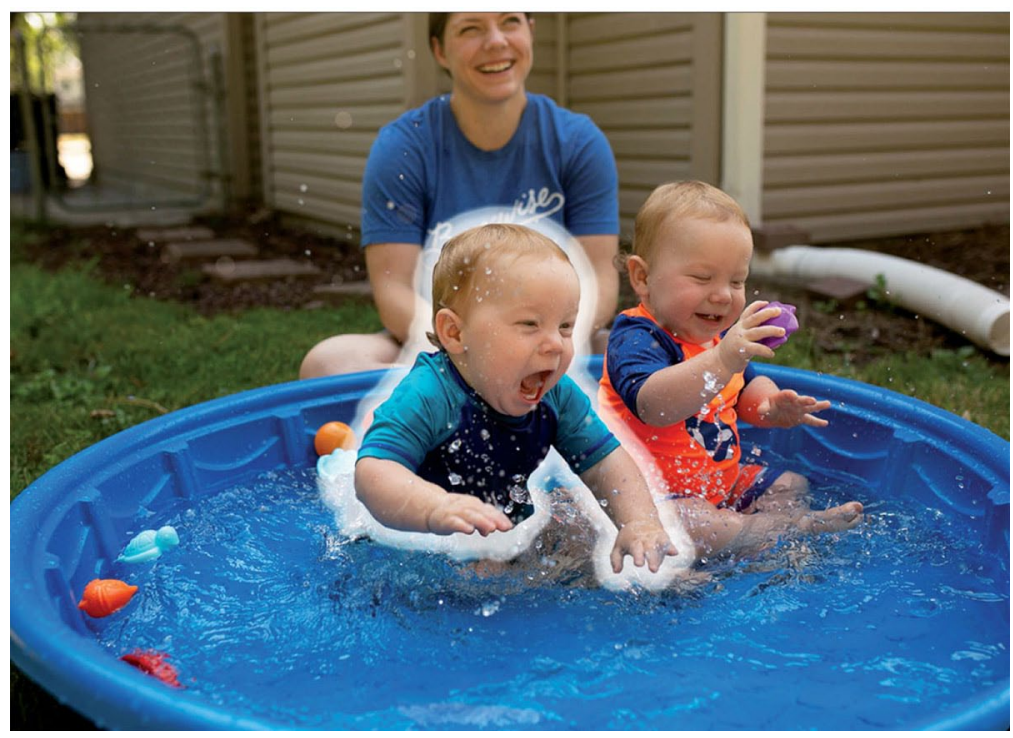

Figure 2. A visual scene display image with the central child is highlighted through increasing the brightness of the background to create a halo like effect.

interest, similar to a halo of edge glow effect (Figure 2), may also help draw the viewers' attention. The concept of background intensification was clearly described by one participant, who explained; 


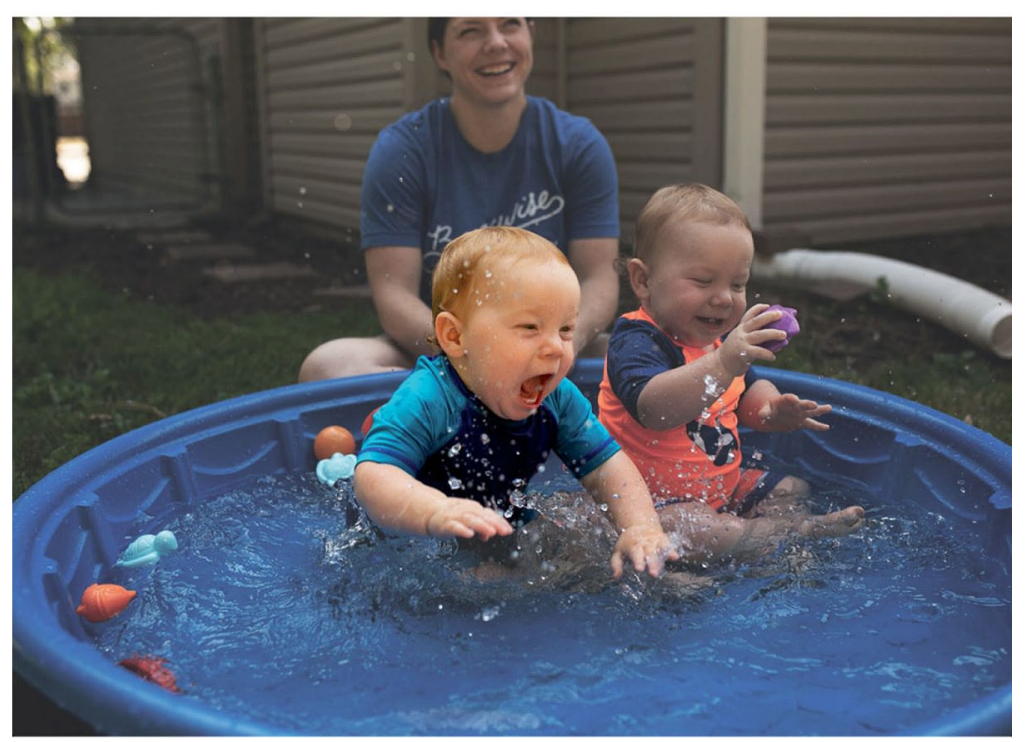

Figure 3. A visual scene display image where the central child is highlighted by saturating target item color and desaturating the background color.

"Well, a drop shadow is probably enough, but you could probably outline it with like something very light and then that kind of pulls it away from in the background. Kind of like a halo around it."

In a parallel fashion, it was also noted that a brief or pulsing flash or luminance change around the object may draw attention to scene objects.

\section{Use of contrast (color)}

In addition to creating contrast through changes in light and luminance, seven participants described the application of color contrast to item highlighting. In more detail, four participants explained how the target item could be made more saturated or vibrant relative to the background which may become more muted (Figure 3), with one expert describing:

"if you've kind of muted everything out and then one thing comes into full color and then back out." In a similar manner, two participants indicated that a black and white background 


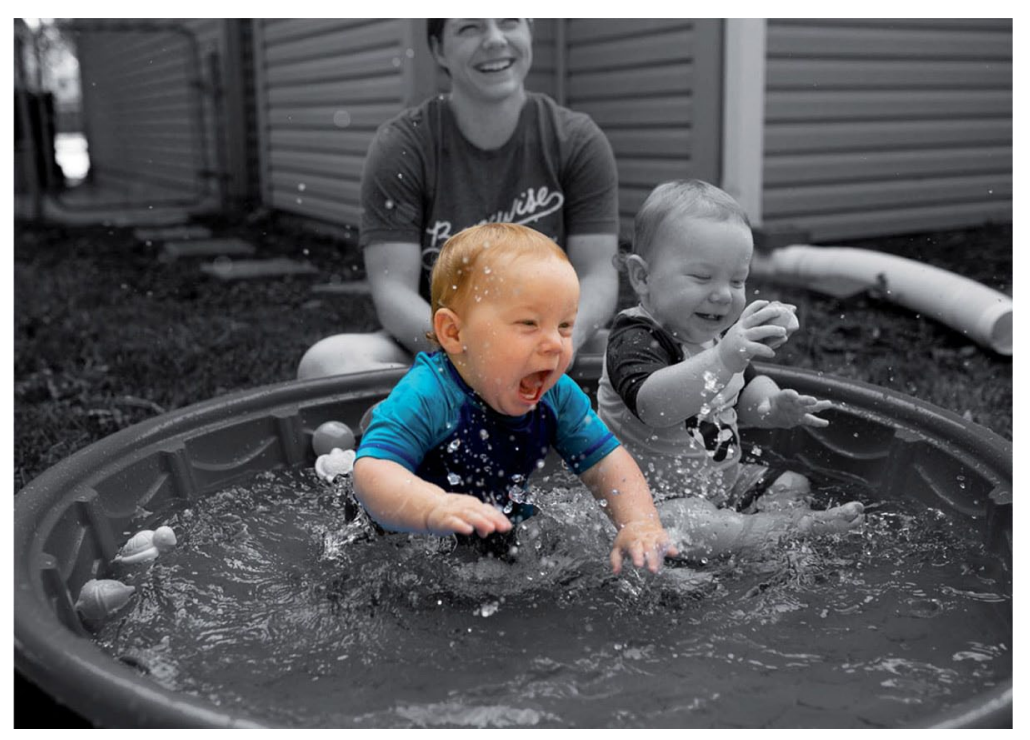

Figure 4. A visual scene display image where the central child is highlighted by making the background black and white.

could be used, with items highlighted in their natural color (Figure 4). Finally, P7 noted that a semitransparent color overlay such as a transparent green shape placed over the target item may help guide attention to the focal object. However, they also noted that a bolded outline may be a clearer method (Figure 5).

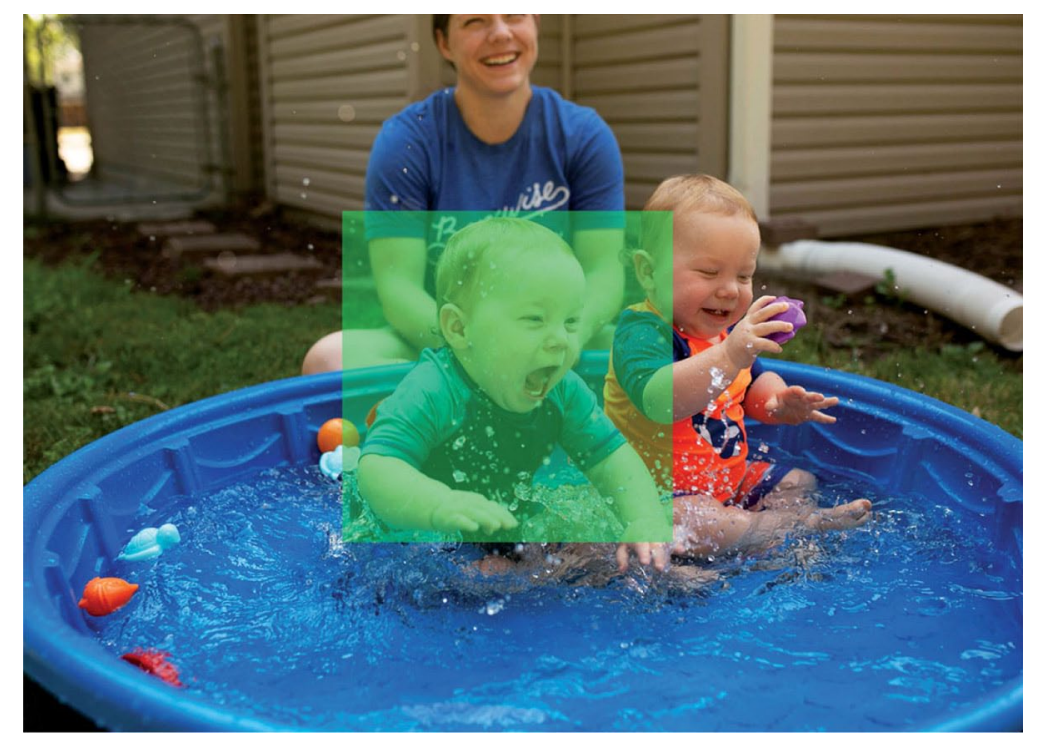

Figure 5. A visual scene display image where the central child is highlighted by overlaying a colored shape (i.e., a green box). 


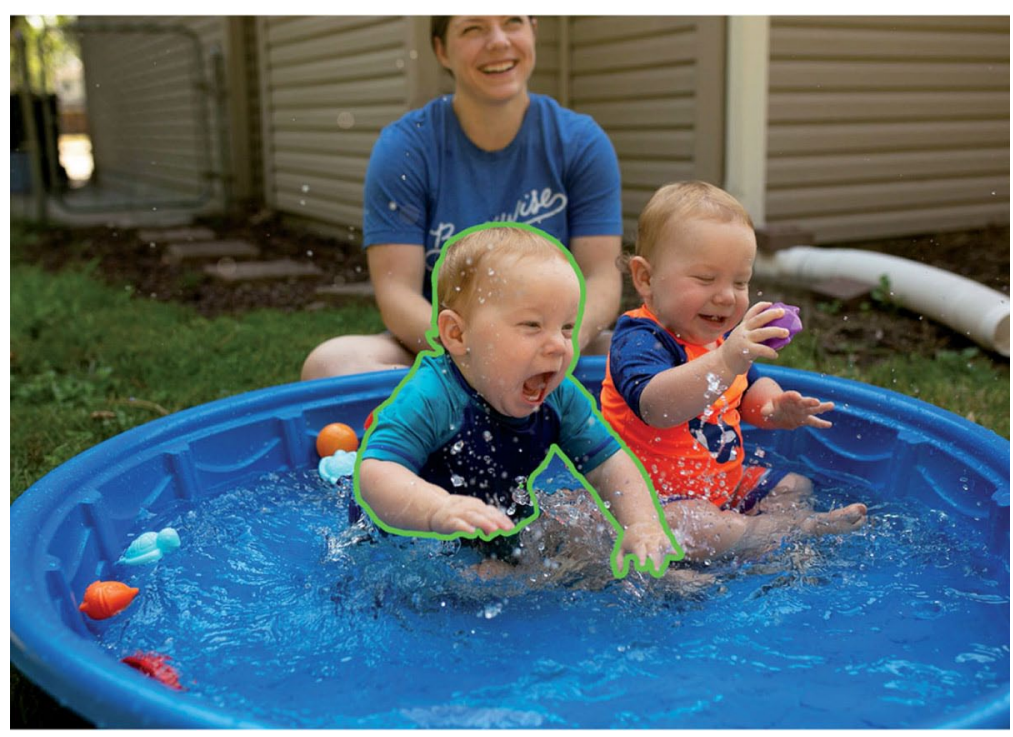

Figure 6. A visual scene display image where the central child is highlighted by a green border outline.

\section{Outline highlighting}

Nine participants discussed using border or outline highlighting, such as a bolded outline around the focal item in a contrasting or more vibrant color than the background, may help draw attention to the target item (Figure 6). However, it was also mentioned that outline highlighting with color may add complexity to the image. For instance, P7 discussed:

"I actually thought that the (i.e., the green outline border used by McCarthy and Boster [1], an image of which was included in our VSD presentation), was pretty effective. It really isolated, specifically the object, um, I thought that was pretty visually clear, I mean, I'm not sure if that's something that is more difficult. I mean, I guess."

In addition, P9 said,

"So the example you, you have here the highlight is a green line outlining everything. And that, that adds confusion for me, watching it." 
Further, two participants noted that consideration needs to be given to the choice of color used for outline highlighting, as some individuals may have impairments in color vision. Finally, two participants noted that the border color could change to provide feedback when a selection is made. For example, P8 said:

"The classic, the classic way of making that interface would be that selection would be in red. And then when you choose to click on that would, it would quickly shift to green. So that the, the user would know that they've made a selection".

\section{Scale and motion}

Three participants noted the use of scale, such as having the item move forward in the visual plane or zooming in on the item to make it larger, may support target recognition (Figure 7). When discussing motion and scale highlighting methods, P4 described:

"When it scans the clock, the clock actually moves forward, like in, in the visual plane, and so then you can see, oh, that's the thing. And then it can zoom back out".

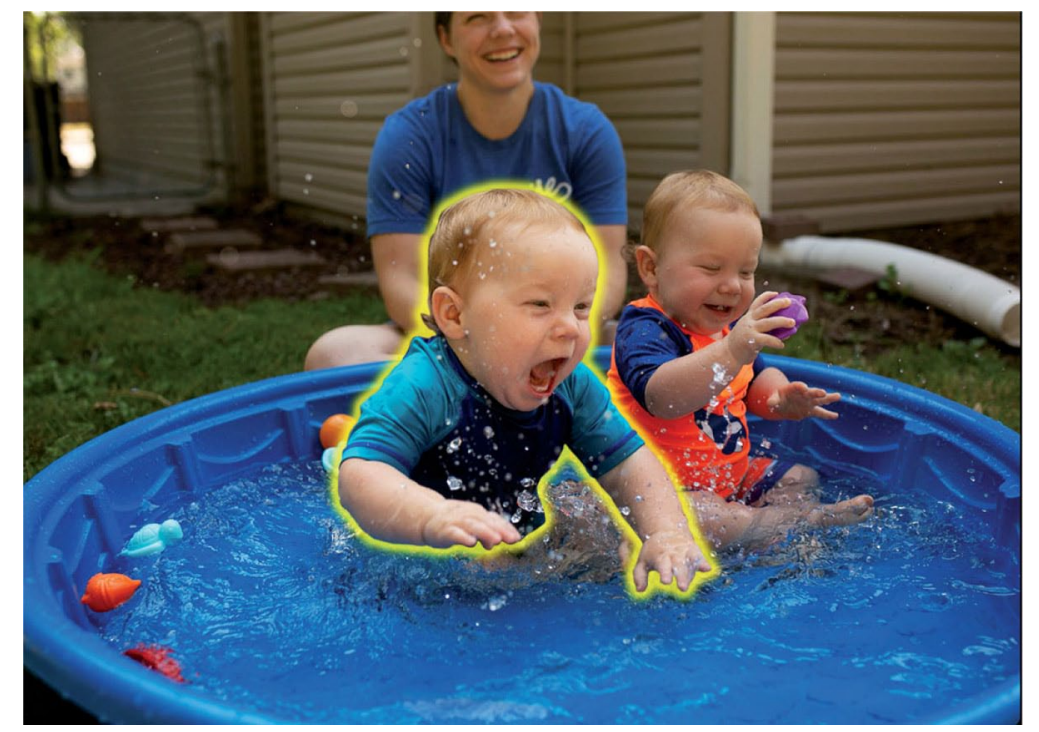

Figure 7. A visual scene display image where the central child is highlighted by zooming/scale in addition to a colored halo effect to illustrate the use of scale and a possible combination highlighting method. 


\section{Discussion and clinical applications}

Study findings provide avenues for using principles of image composition to draw attention to target items in a VSD display during item scanning and highlighting. Identified themes are consistent with artistic and photographic principles of composition that may help support attention to focal objects, detailing techniques such as use of light and color contrast, outline highlighting, in addition to scale [2]. Further, findings also parallel existing research identifying how composition principles, such as scale, can improve scanning performance [16]. However, results extend existing findings to provide a framework for how a variety of composition principles can be applied to item scanning within VSDs. The following discussion provides a summary of the identified subthemes in relation to existing AAC research, in addition to how findings can be applied to JIT programming for VSDs, and $\mathrm{BCI}$ technology for $\mathrm{AAC}(\mathrm{BCI}-\mathrm{AAC})$.

\section{Light and color contrast}

The use of light and color contrast is a strong method that may help automatically draw our attention in scenes $[2,32]$. Therefore, participants provided an array of options for maximizing color and light contrast in drawing attention to highlighted scene elements. Findings parallel techniques commonly used to draw individuals' attention in the everyday world, photography, and cinema. For instance, regarding light contrast, participants described making the object brighter relative to the background, like a spotlight or drop shadow effect, or making the background lighter relative to the focal object, making the object look like it has a halo. Naturally, light contrast can help provide a strong outline around focal objects [2], and may help objects stand out by increasing their level of salience. More specifically, scene areas that are not uniform along salient features such as luminance, contrast, and color are identified as more informative than their uniform scene counterparts [33]. Therefore, increasing an object's salience may help with engagement in highlighted AAC items.

Increasing the color saturation of an item relative to its background may also help an item to stand out. For example, participants discussed that an item could become more colorful or saturated in 
contrast to the background, which may become more muted during highlighting. Furthermore, the highlighted item could remain in its natural color while the background became black and white. The concept of increasing color contrast by providing a black and white background parallels composition techniques used by popular movies such as Schindler's List, which highlights a girl in a red coat on a black and white backdrop [34] or Pleasantville where color splashes were used in black and white scenes [35]. The provision of color contrast is well documented as a strong attractor of attention in scenes [36]. Therefore, it provides a logical avenue for engaging individuals in highlighted scene elements. However, it should be considered that scenes including color may support information recall in contrast to scenes presented in black and white $[13,37]$. Therefore, while more research is needed, keeping scene color during the offer stage of item scanning may help support recall. It is possible, however, that the effect could still be useful for providing feedback after item selection. The purpose of feedback on selection is to highlight the specific chosen items and set them apart from the rest of the field. For instance, in McCarthy et al. [16], feedback was provided by the selected item moving into the middle of the screen and becoming enlarged to the extent it obscured neighboring choices.

Finally, the utilization of non-natural color overlays (e.g., a green shape overlay) was also expressed by one participant. However, they believed this technique may be less effective than outline highlighting, possibly due to how people naturally perceive the importance of objects in nature through contrast [2]. Further, it should be considered that VSDs may be captured in a variety of settings; thus, future investigations should seek to evaluate how different environments impact the effectiveness of highlighting techniques. For instance, if an image is taken on a sunny day with high levels of light contrast or on a bright white background (e.g., a bright white blanket), then the visual impact of increasing the lightness of the background to create a halo effect may be limited, so another option (e.g., color contrast, zoom, outline highlighting) may serve to better engage attention. 


\section{Outline highlighting, scale and motion}

Outline or border highlighting is a commonly used strategy for highlighting items within a grid display. For instance, in a grid display, each cell may be highlighted by a red square [16] and in a contextual scene the object or person within a scene can be outlined [1]. The bolded outlines drawn around items in visual scenes can be made visible for feedback on selection purposes in many apps. Therefore, study findings continue to support existing clinical practice that border highlighting may help isolate the focal cell/item. However, border highlighting methods may also add a level of complexity, possibly due to the abstract nature of border highlighting given that we do not present or highlight important focal elements in nature through a bold color outline [16]. Figure 7. A visual scene display image where the central child is highlighted by zooming/scale in addition to a colored halo effect to illustrate the use of scale and a possible combination highlighting method. Further, our experts noted that an individual's color vision needs to be considered when utilizing a color border highlighting.

In addition to border highlighting, some existing AAC devices also provide scale and zoom highlighting options to support access to grid displays. When an object is of increased size or scale in addition to its surroundings, it is perceived as being more important [2]. Thus, in addition to adding importance to the focal object, the foreground movement parallels how individuals may receive an item in their natural environment (e.g., they receive the object given), possibly decreasing learning demands. Motion is a strong attractor of attention $[8,38]$, and utilizing simple motion (e.g., an item gets bigger or smaller, spins, moves up and down, or transverse) may help support individual attention to communication items within the AAC display $[8,38]$. Thus, our findings continue to support the use of simple motion, which were important components in the enhanced scanning paradigm developed by McCarthy et al. [16]. In more detail, in comparison to the traditional border highlighting method of grid items, McCarthy et al. [16] attempted to lower the learning demands of scanning-based AAC access for 2-year-olds without disabilities. Their enhanced scanning system presented (highlighted) grid items via a small increase in scale, with the item dramatically increasing its scale, as if 
the child were actually receiving the item upon selection, finding improved performances for their enhanced scanning display.

In addition to simple motion, complex motion (e.g., the hands on a clock move when highlighted [38]) can increase the selection accuracy of VSD items for 2.5- to 3.5-year-olds without disability, in contrast to outline highlighting [1]. Therefore, in addition to simple motion, complex motion may be an important consideration in scanning-based VSD design. However, while our experts noted the importance of scale and zoom (simple motion strategies), complex motion was not discussed. The lack of discussion regarding complex motion by our experts may be due to the focus of the discussion surrounding composition principles for static 2D photographic images. Therefore, while scale plays a role in static image design, complex motion may not have been considered by our experts as an option. Thus, while the role of complex motion in VSD highlighting requires further attention, how complex motion highlighting can be applied to photographic images especially in a JIT scenario requires further thought. However, complex motion may be readily utilized to support VSD scanning access to programmed or computer-generated VSDs and should be considered in AAC development.

\section{Considerations for AAC development to support JIT programming of VSDs}

JIT is an important component to support VSD access and communication in random or spontaneous situations. JIT programming for VSDs focuses on developing software that allows for quick image capture and easy programming to take maximum advantage of the individual's immediate environment/context to help maximize communication success [9]. Therefore, how to quickly apply highlighting methods to VSD is paramount to support active communication in the environment/event the individual is currently participating. As described above, commercial AAC displays may already provide automatic options for item scanning in grids, such as zooming and border highlighting. However, the application of automatic highlighting methods to VSDs, especially photographs, in a JIT scenario remains unexplored. Currently, JIT programming software for VSDs may allow an individual to capture a photo and draw a selection area which 
can be utilized as a communication hotspot [39]. Therefore, paralleling existing JIT programming methods, VSDs may benefit from utilizing artificial intelligence (Al) or machine learning methods to support object identification, labelling, and the provision of relevant text, as appropriate [40]. Scanning order could be done by recording the desired scanning in order and having that recording playback. Existing screen recording and customization platforms can do this with a variety of animated content (e.g., Tumult HypeVR) [41].

Applying software-utilizing Al or machine learning methods to VSDs may be especially helpful to JIT program VSDs for scanningbased access, since identifying object borders accurately may best support application of outlined highlighting principles to scene objects. Thus, AAC developers may wish to consider utilizing new Albased applications similar to those found in Adobe Photoshop (Adobe Systems, San Jose, CA). For instance, recent Photoshop tools such as Select Subject and Object Selection allow for quick selection of scene objects the individual wishes to identify. In more detail, by using the Select Subject tool, the software can automatically select scene element(s) identified as the most important with as little as one click from the operator. However, if the individual wishes to control the selection of scene element(s), by using the Object Selection tool they can drag a box over target item(s) or provide a rough outline around them. Following the rough identification of the object by the operator, the software then provides automatic selection of targeted element(s) [42]. Developing AAC devices that support quick and automatic selection of scene elements for rapid programming will help individuals using AAC and their support network to quickly identify target elements, apply relevant vocabulary, and apply preprogrammed highlighting techniques. Further, once the objects are selected, a variety of effects like color saturation or other animated movement could be applied as feedback on selection. Feedback can be an individual animation that plays since no interaction is required with the feedback. For instance, video VSDs involve embedded video that plays after selection of items [43]. However, while new object detection software advancements show promise, further testing is needed regarding object recognition in photographs used for VSDs where object borders may be unclear. 


\section{Application of highlighting principles to $B C I-A A C$ and eye-gaze technology}

While the focus of the interviews was scanning-based AAC access, study findings may also inform the development of P300-based braincomputer interfaces for AAC (BCl-AAC), and eye-gaze access. Specifically, P300-based $\mathrm{BCl}-\mathrm{AAC}$ designs also utilize item highlighting for AAC access. In more detail, the P300 event-related potential (brain signal) is produced when the brain processes a visual, auditory, or tactile stimulus that is novel. Therefore, to access a visually based P300based $\mathrm{BCl}-\mathrm{AAC}$ device, the individual focuses their attention of the specific target communication element they wish to select (e.g., a letter or symbol in a grid) [44]. While focusing on the target communication element, all items within the display are then randomly highlighted (e.g., by repeatedly changing from grey to white) [45]. During this period, a positive deflection in the electroencephalography signal is present approximately $300 \mathrm{~ms}$ after the novel target item is flashed, relative to the frequent non-target items. After multiple target flashes, the presence of the P300 triggers selection of the target item $[44,46]$.

Existing P300-based BCl-AAC research has already begun evaluating concepts identified in this study to grid-based displays access with positive results. For instance, to inform P300-based BCl-AAC design, research has begun evaluating how factors such as light and color contrast $[47,48]$ and motion impact BCl-AAC outcomes [49]. For instance, highlighting items in a spelling grid display with increased luminosity/brightness (i.e., highlighting letters in a bright white flash on a black background, versus a less bright flash) was rated preferential and boosted the $\mathrm{BCl}-\mathrm{AAC}$ performance of neurotypical individuals and those with motor impairments [47]. Further, in contrast to a grey-towhite flash, the use of color to highlight items, such as turning a letter from green to blue when highlighted [50] may improve P300-based $\mathrm{BCl}-\mathrm{AAC}$ performance by enhancing brain activity in parietal and occipital cortices, which are involved in P300 generation [51]. However, while research is limited, it may possibly be more beneficial to flicker between colors (e.g., going from a grey to a color) than to alter color saturation (e.g., altering a letter from a dark red to a light red [52]. Finally, simple motion techniques, such as zoom where a letter increased in font size by 5 when highlighted [48] or moving the letter from left 
to right when combined with a colored flash [49], may improve $\mathrm{BCl}$ AAC accuracy. However, as existing P300-based $\mathrm{BCl}-\mathrm{AAC}$ highlighting techniques have been minimally applied to scenes, study findings extend current research by providing a framework for investigation of how composition factors impact highlighting of scene elements for P300 BCl-AAC purposes [2].

In relation to eye-gaze AAC access, current commercial AAC devices commonly provide feedback regarding where AAC device is interpreting the individual's gaze location to be placed upon the screen. For instance, in addition to a dot in indicating gaze location, for a grid display, the grid item currently being viewed by the AAC user may change in background color, be border-highlighted, or both [53]. The feedback provided by the eye-gaze software to the individual can help facilitate AAC success [54]. Therefore, paralleling current commercial procedures for eye-gaze access, the compositional principles outlined in this paper may also be utilized to provide feedback regarding which hotspot the AAC software is currently identifying as being gazed upon by the individual to support VSD access via eye gaze. However, consideration needs to be given to ensuring feedback does not detract an individual's gaze from primary focal elements, possibly by only highlighting single elements at any given time, as detraction may negatively impact AAC success.

\section{Limitations}

Several limitations should be considered with regard to this study. Specifically, the investigation only assessed perspectives regarding how to engage an individual's attention to highlighted elements using composition principles. However, engaging the individual in the AAC display is only one piece of the scanning puzzle. For instance, for successful scanning-based AAC access, the individual must also be able to time their selection [3], and also make an abstract association between a button press (e.g., by moving their head) and a device selection [16]. Further, participants only had a limited introduction to AAC and scanning. The idea of direct selection on a touchscreen may be more intuitive than scanning. Therefore, ideally participants would have had an opportunity to try scanning themselves and/or to meet 
a person who uses the technique. In addition to giving composition experts scanning experience, a broader set of input on possibilities from the perspectives of individuals with expertise in human computer interaction for children or developmental psychology may better inform how these highlighting principles can be utilized to lower the overall learning demands associated with scanning access. Further, the study included a limited number of experts, and did not include the important viewpoints of those with complex communication needs or their support network, which is an important component to AAC development.

\section{Future directions}

Study data provided insights into how photographic composition can be utilized for highlighting VSD elements. However, further research is required to inform AAC development. Access techniques need to be accurate, efficient, and minimally fatiguing [3]. In addition to identifying performance outcomes (e.g., accuracy) associated with different highlighting techniques for those with complex communication needs due to varying aetiologies, it is possible some individuals may find different highlighting techniques more appealing and/or less fatiguing. Furthermore, as children may have different preferences to adults [55], how an individual's unique preferences may change across the life span or disease course should be considered when evaluating highlighting principles identified through this study. Therefore, identifying the perspectives of those with complex communication needs and their support network needs to be considered alongside performance measures.

In an even broader sense, the extent to which a scanned VSD takes an individual "out of" an interaction should be considered. VSDs are meant to map directly on to a scene to facilitate interactions and in many cases to be able to repeat these actions to build a sense of anticipation and routine [56]. Thus, further study is required to know the extent to which scanned VSDs support interactions in a comparable way to direct selection ones. For instance, should there be a lack of connection or interaction, or should the turn exchange rate be too slow using scanning access to facilitate meaningful communication, 
then other techniques should be considered, such as partner-assisted scanning or modelling of scanning by partners, to support communication interactions.

Another important consideration for future research is that study highlighting techniques were primarily discussed by our experts in isolation. However, future research should also consider the combination of highlighting techniques (e.g., zooming while increasing light and/or color contrast; Figure 7). For instance, Jin et al. [52] found that $\mathrm{P} 300$-based $\mathrm{BCl}-\mathrm{AAC}$ performance increased when simple linear motion and colored flash were combined, in comparison to each technique in isolation. Further, McCarthy et al. [16] noted the importance of considering the object "offer" (highlighting) in addition to selection feedback, which could utilize different methods to increase stimulus saliency and limit learning demands. It may be intuitive to highlight VSD elements in a sequential/linear order using the same highlighting technique [1]. However, the scanning pattern used in grid displays (e.g., row-column scanning versus linear scanning) may also impact cognitive load [57]. Therefore, the pattern/order of item highlighting may require complementary consideration along with how the object is highlighted. For instance, scenes may include objects that are more likely to be primary focal elements for communication selection versus secondary or more background elements. Therefore, future research may wish to consider the combined impacts of the highlighting methods and pattern. For example, future research for scanning-based VSD access may seek to evaluate scanning patterns that highlight primary focal elements first and secondary elements second, using the same or different highlighting methods, to lower cognitive burdens associated with scanning access. Further, one expert noted that a linear/ predictable scanning pattern similar to traditional AAC methods may lower cognitive demands.

Finally, AAC access for individuals with visual impairments may be supported by auditory scanning methods [58] along with AAC display changes to provide large icons or greater contrast levels [59]. Therefore, future research is needed to see our discussed highlighting principles may support AAC success for those with visual impairments. For example, individuals with cortical visual impairment (CVI) may have specific color preferences while other individuals could have a more limited range of color discrimination [60]. Therefore, procedures for 
customizing a color palette based on an assessment of preferences could be highly valuable in selecting highlighting color. Further, as many or our identified approaches focus on increase contrast, future research may identify which proposed methods of highlighting are most beneficial for those with visual impairments. Beyond the visual interface alone, individuals with CVI may have visual fixations that are much briefer than an individual without a visual impairment meaning that an additional auditory cue could help draw focus [61]. Thus, using multiple sensory modalities for AAC interfaces with individuals with CVI and complex communication needs is supported by the literature, however despite the promise of auditory enhancements there has been little research in that area [60]. Paralleling visual methods, how an item is auditorily highlighted (e.g., by producing an associated sound or word, or providing directional cues, each time an item is highlighted) may impact scanning- based [58] and auditorybased P300-based $\mathrm{BCl}-\mathrm{AAC}$ performance [62]. An understanding of psychoacoustic principles and how they interact with visual stimuli is an important intersection to study in building complementary auditory and visual interfaces [63]. Roman-Lantzy stated "CVI is a disability of access - access to the visual world" [61,p.v]. Therefore, to ensure inclusive AAC design, auditory highlighting techniques should also be considered, along with how different visual highlighting techniques may support those with visual impairments by adapting factors such as item size and contrast levels.

Acknowledgments The authors wish to thank Kyleigh Skaggs and Seth Teager for figure development, along with Megan Salley, Marj McKinty, Michaela Reddel, Hannah Smith, and Austin Spoor for their contributions to the project.

Disclosure statement The authors report no potential competing interests.

Funding This work was supported by the Nebraska Tobacco Settlement Biomedical Research Development Fund. 


\section{References}

1 ) McCarthy J, Boster J. A comparison of the performance of 2.5 to 3.5-year-old children without disabilities using animated and cursor-based scanning in a contextual scene. Assist Technol. 2018;30(4):183-190.

2 ) Pitt K, McCarthy J. What's in a photograph? The perspectives of composition experts on factors impacting visual screen display complexity for augmentative and alternative communication, and strategies for improving visual communication. Am J Speech Lang Pathol. 2021;30(5): 2080-2097.

3 ) Beukelman DR, Light JC. Augmentative and alternative communication: supporting children and adults with complex communication needs. 5th ed. Baltimore (MD): Paul H. Brookes Publishing; 2020.

4 ) Boster JB, McCarthy JW. When you can't touch a touch screen. Semin Speech Lang. 2017;38(4):286-296.

5 ) McCarthy JW, Boster JB. Growing up with technology: does the device go in the Middle? Top Lang Disord. 2019;39(4): E1-E16.

6 ) Wilkinson KM, Jagaroo V. Contributions of principles of visual cognitive science to AAC system display design. Augment Altern Commun. 2004;20(3):123-136.

7 ) Light J, Wilkinson KM, Thiessen A, et al. Designing effective AAC displays for individuals with developmental or acquired disabilities: state of the science and future research directions. Augment Altern Commun. 2019;35(1): 42-55.

8 ) Thiessen A, Thistle J, Brown J. Clinical and research perspectives on visual scene displays. Am J Speech Lang Pathol. 2021;30(3):1134-1156.

9 ) Holyfield C, Caron J, Light J. Programing AAC just-in-time for beginning communicators: the process. Augment Altern Commun. 2019;35(4):309-318.

10 ) Caron J, Light J, Davidoff BE, et al. Comparison of the effects of mobile technology AAC apps on programming visual scene displays. Augment Altern Commun. 2017;33(4): 239-248.

11 ) Light J, McNaughton D, Caron J. New and emerging AAC technology supports for children with complex communication needs and their communication partners: state of the science and future research directions. Augment Altern Commun. 2019;35(1):26-41.

12 ) Beukelman DR, Hux K, Dietz A, et al. Using visual scene displays as communication support options for people with chronic, severe aphasia: a summary of AAC research and future research directions. Augment Altern Commun. 2015; 31(3):234-245.

13 ) Ratcliff A. Comparison of relative demands implicated in direct selection and scanning: considerations from normal children. Augment Altern Commun. 1994;10(2):67-64.

14 ) Petersen K, Reichle J, Johnston SS. Examining preschoolers' performance in linear and row-column scanning techniques. Augment Altern Commun. 2000;16(1):27-36.

15 ) Light J, Lindsay P. Cognitive science and augmentative and alternative communication. Augment Altern Commun. 1991; 7(3):186-203. 
16 ) McCarthy J, Light J, Drager K, et al. Re-designing scanning to reduce learning demands: the performance of typically developing 2-year-olds. Augment Altern Commun. 2006; 22(4):269-283.

17 ) Wilkinson KM, Light J. Preliminary investigation of visual attention to human figures in photographs: potential considerations for the design of aided AAC visual scene displays. J Speech Lang Hear Res. 2011;54(6):1644-1657.

18 ) Wilkinson KM, Light J, Drager K. Considerations for the composition of visual scene displays: potential contributions of information from visual and cognitive sciences. Augment Altern Commun. 2012;28(3):137-147.

19 ) Wilkinson KM, Light J. Preliminary study of gaze toward humans in photographs by individuals with autism, down syndrome, or other intellectual disabilities: implications for design of visual scene displays. Augment Altern Commun. 2014;30(2):130-146.

20 ) Fager SK, Fried-Oken M, Jakobs T, et al. New and emerging access technologies for adults with complex communication needs and severe motor impairments: state of the science. Augment Altern Commun. 2019;35(1):13-25.

21 ) Pitt KM, Brumberg JS, Pitt AR. Considering augmentative and alternative communication research for brain-computer interface practice. Assist Technol Outcomes Benefits. 2019; 13(1):1-20. Summer

22 ) Pitt KM, Brumberg JS. Guidelines for feature matching assessment of BrainComputer Interfaces for Augmentative and Alternative Communication. Am J Speech Lang Pathol. 2018;27(3):950-964.

23 ) Hajjar DJ, McCarthy JW, Benigno JP, et al. "You get more than you give": experiences of community partners in facilitating active recreation with individuals who have complex communication needs. Augment Altern Commun. 2016; 32(2):131-142.

24 ) Saunders B, Sim J, Kingstone $T$, et al. Saturation in qualitative research: exploring its conceptualization and operationalization. Qual Quant. 2018;52(4):1893-1907.

25 ) NVivo [Internet]. Melbourne (Australia): QSR International; 2021; [cited 2021 May 7]. Available from: https://www.qsrinternational.com/nvivo/products .

26 ) Gibbs GR. Analyzing qualitative data. New York (NY): Sage Publications; 2008.

27 ) Creswell J. Qualitative inquiry and research design. 3rd ed. London: Sage Publications; 2013.

28 ) O'Neill T, Wilkinson K. Preliminary investigation of the perspectives of parents of children with cerebral palsy on the supports, challenges, and realities of integrating augmentative and alternative communication into everyday life. Am J Speech Lang Pathol. 2020;29(1):238-254.

29 ) Syed M, Nelson S. Guidelines for establishing reliability when coding narrative data. Emerg Adulthood. 2015;3(6): 375-387.

30 ) McHugh ML. Interrater reliability: the kappa statistic. Biochem Med. 2012;22(3):276-282.

31 ) Brantlinger E, Jimenez R, Klingner J, et al. Qualitative studies in special education. Except Child. 2005;71(2):195-207. 
32 ) Turatto M, Galfano G. Color, form, and luminance capture attention in visual search. Vision Res. 2000;40(13): 1639-1643.

33 ) Henderson JM, Hayes TR. Meaning guides attention in real-world scene images: evidence from eye movements and meaning maps. J Vis. 2018;18(6):10.

34 ) Doherty T. Review: [untitled]. Cinéaste. 1994;20(3):49-51.

35 ) Dickinson G. The Pleasantville Effect: nostalgia and the visual framing of (White) suburbia. West J Commun. 2006; 70(3):212-233.

36 ) Wolfe JM, Horowitz TS. Five factors that guide attention in visual search. Nat Hum Behav. 2017;1(3):1-8.

37 ) Wichmann F, Sharpe L, Gegenfurtner K. The contributions of color to recognition memory for natural scenes. J Exp Psychol Learn Mem Cogn. 2002;28(3):509-520.

38 ) Jagaroo V, Wilkinson K. Further considerations of visual cognitive neuroscience in aided AAC: the potential role of motion perception systems in maximizing design display. Augment Altern Commun. 2008;24(1):29-42.

39 ) Tobii Dynavox Snap Scene: user's manual. [Stockholm (Sweden)]: Tobii AB; 2016; [cited 2021 Aug 31]. Available from: http://tdvox.web-downloads. s3.amazonaws.com/SnapScene/documents/TobiiDynavox SnapScene UserManual v1.2 en-US.pdf.

40 ) Sennott S, Akagi L, Lee $M$, et al. AAC and artificial intelligence (AI). Top Lang Disord. 2019;39(4):389-403.

41 ) Tumult Hype 4.1 [Internet]. San Francisco (CA): Tumult; 2020; [cited 2021 May 8]. Available from: https://tumult.com/hype/.

42 ) Clark P. Photoshop: Now the world's most advanced Al application for creatives [Internet]. Adobe Blog; 2020; [cited 2021 May 8]. Available from: https://https://blog.adobe.com/en/publish/2020/10/05/photoshop-now-theworlds-most-advanced-ai-application-for-creatives.html\#gs.8j5ty1 .

43 ) Babb S, McNaughton D, Light J, et al. Using AAC video visual scene displays to increase participation and communication within a volunteer activity for adolescents with complex communication needs. Augment Altern Commun. 2020;36(1):31-42.

44 ) Brumberg JS, Pitt KM, Mantie-Kozlowski A, et al. Brain-computer interfaces for augmentative and alternative communication: a tutorial. Am J Speech Lang Pathol. 2018;27(1): 1-12.

45 ) Donchin E, Spencer K, Wijesinghe R. The mental prosthesis: assessing the speed of a P300-based brain-computer interface. IEEE Trans Rehabil Eng. 2000;8(2):174-179.

46 ) Pitt KM, Brumberg JS, Burnison JD, et al. Behind the scenes of noninvasive brain-computer interfaces: a review of electroencephalography signals, how they are recorded, and why they matter. Perspect ASHA Spec Interest Groups. 2019;4(6):1622-1636.

47 ) Li Y, Bahn S, Nam C, et al. Effects of luminosity contrast and stimulus duration on user performance and preference in a P300-based brain-computer interface. Int J Hum Comput Interact. 2014;30(2):151-163. 
48 ) Gibert G, Attina V, Mattout J, et al.. Size enhancement coupled with intensification of symbols improves P300 speller accuracy. In: Müller-Putz GR, Brunnenr C, Leeb R, Pfurtscheller G, Neuper C, editors. Proceedings of the 4th International BCI Workshop and Training Course. Graz (Austria): Verlag der Technischen Universität Graz; 2008. p. 250-255.

49 ) Jin J, Allison B, Wang $X$, et al. A combined brain-computer interface based on P300 potentials and motion-onset visual evoked potentials. J Neurosci Methods. 2012;205(2): 265-276.

50 ) Takano K, Komatsu T, Hata N, et al. Visual stimuli for the P300 brain computer interface: a comparison of white/gray and green/blue flicker matrices. Clin Neurophysiol. 2009;120(8):1562-1566.

51 ) Ikegami S, Takano K, Wada M, et al. Effect of the green/ blue flicker matrix for P300-based brain-computer interface: an EEG-fMRI study. Front Neurol. 2012;3:113.

52 ) Ryan D, Townsend G, Gates N, et al. Evaluating brain-computer interface performance using color in the P300 checkerboard speller. Clin Neurophysiol. 2017;128(10): 2050-2057.

53 ) Prentke Romich Company. NuEye ${ }^{\mathrm{TM}}$ Tracking System: user's guide. Wooster (OH): Prentke Romich Company; 2012; Available from: https:// cpb-ca-c1.wpmucdn.com/learningnetwork.setbc.org/dist/5/501/ files/2017/05/16440v1.4-NuEye-Usr-Guide-1y8vtvm.pdf.

54 ) Chen S, O'Leary M. Eye Gaze 101: What speech-language pathologists should know about selecting eye gaze augmentative and alternative communication systems. Perspect ASHA Sigs. 2018;3(12):24-32.

55 ) Light J, Page R, Curran J, et al. Children's ideas for the design of AAC assistive technologies for young children with complex communication needs. Augment Altern Commun. 2007;23(4):274-287.

56 ) Light J, McNaughton D. Designing AAC research and intervention to improve outcomes for individuals with complex communication needs. Augment Altern Commun. 2015; 31(2):85-96.

57 ) Thistle J, Wilkinson K. Working memory demands of aided augmentative and alternative communication for individuals with developmental disabilities. Augment Altern Commun. 2013;29(3):235-245.

58 ) McCarthy J, DiGiovanni J, Ries D, et al. Exploration of head related transfer function and environmental sounds as a means to improve auditory scanning for children requiring augmentative and alternative communication. Assist Technol. 2020;32(6):325-334.

59 ) Batorowicz B, McDougall S, Shepherd TA. AAC and community partnerships: the participation path to community inclusion. Augment Altern Commun. 2006;22(3):178-195.

60 ) Boster JB, McCarthy JW, Brown K, et al. Creating a path for systematic investigation of children with cortical visual impairment who use augmentative and alternative communication. Am J Speech Lang Pathol. 2021;30(4):1880-1893. 
61 ) Roman-Lantzy C. Cortical visual impairment: an approach to assessment and intervention. 2nd ed. New York (NY): AFB Press; 2018.

62 ) Käthner I, Ruf CA, Pasqualotto E, et al. A portable auditory P300 brain-computer interface with directional cues. Clin Neurophysiol. 2013;124(2):327-338.

63 ) DiGiovanni JJ, Riffle TL, McCarthy JW. Auditory stroop using spatial stimuli. Clin Arch Commun Disord. 2017;2(2): 178-184. 